\title{
Design and expected performances of the large acceptance calorimeter for the HERD space mission
}

Lorenzo Pacini, ${ }^{\mathrm{a}, *}$ Oscar Adriani, ${ }^{\mathrm{a}, \mathrm{b}}$ Yong-lin Bai, ${ }^{\mathrm{c}}$ Tian-wei Bao, ${ }^{\mathrm{d}}$ Eugenio Berti, ${ }^{\mathrm{a}, \mathrm{b}}$ Sergio Bottai, ${ }^{\mathrm{a}}$ Wei-wei Cao, ${ }^{\mathrm{c}}$ Jorge Casaus, ${ }^{\mathrm{h}}$ Xing-zhu Cui, ${ }^{\mathrm{d}}$ Raffaello D’Alessandro, ${ }^{\mathrm{a}, \mathrm{b}}$ Valerio Formato, ${ }^{\mathrm{i}}$ Jia-rui Gao, ${ }^{\mathrm{c}}$ Ran Li, ${ }^{\mathrm{c}}$ Xin Liu, ${ }^{\mathrm{d}}$ Leonarda Lorusso, ${ }^{\mathrm{e}}$ Lin-wei Lyu, ${ }^{\mathrm{c}}$ Jesus Marin, ${ }^{\mathrm{h}}$ Gustavo Martinez, ${ }^{\mathrm{h}}$ Cecilia Pizzolotto, ${ }^{1}$ Jun-jun Qin, ${ }^{\mathrm{c}}$ Zheng Quan, ${ }^{\mathrm{d}}$ Da-lian Shi, ${ }^{\mathrm{c}}$ Oleksandr Starodubtsev, ${ }^{\mathrm{a}}$ Zhi-cheng Tang, ${ }^{\mathrm{d}}$ Alessio Tiberio, ${ }^{\mathrm{a}, \mathrm{b}}$ Valerio Vagelli, ${ }^{\mathrm{f}, \mathrm{g}}$ Miguel Angel Velasco, ${ }^{\mathrm{h}}$ Bo Wang, ${ }^{\mathrm{c}}$ Rui-jie Wang, ${ }^{\mathrm{d}}$ Zhi-gang Wang, ${ }^{\mathrm{d}}$ Ming Xu, ${ }^{\mathrm{d}}$ Yang Yang, ${ }^{\mathrm{c}}$ Li Zhang ${ }^{\mathrm{d}}$ and Jin-kun Zheng $^{\mathrm{c}}$ on behalf of the HERD Collaboration

(a complete list of authors can be found at the end of the proceedings)

${ }^{\mathrm{a}}$ INFN sezione di Firenze, I-50019 Sesto Fiorentino, Florence, Italy

${ }^{\mathrm{b}}$ Department of Physics and Astronomy, University of Florence, I-50019 Sesto Fiorentino, Florence, Italy

${ }^{\mathrm{c}} \mathrm{Xi}$ 'an Institute of Optics and Precision Mechanics of CAS, Xi'an, China

${ }^{\mathrm{d}}$ Key Laboratory of Particle and Astrophysics, Chinese Academy of Sciences, Beijing, China

e Dipartimento di Matematica, Università degli Studi di Roma "Tor Vergata", Via Ricerca Scientifica 1, 00133, Roma, Italia

${ }^{\mathrm{f}}$ Agenzia Spaziale Italiana (ASI), I-00133 Roma, Italy

$\mathrm{g}$ Istituto Nazionale di Fisica Nucleare (INFN), Sezione di Perugia, I-06123 Perugia, Italy

${ }^{\mathrm{h}}$ Centro de Investigaciones Energéticas, Medioambientales y Tecnoló gicas (CIEMAT), E-28040 Madrid, Spain

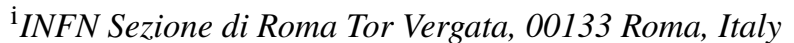

${ }^{1}$ INFN Sezione di Trieste, I-34149 Trieste, Italy

E-mail: lorenzo.pacini@fi.infn.it

\footnotetext{
${ }^{*}$ Presenter
} 
The High Energy cosmic-Radiation Detection (HERD) is a future space experiment which will be installed on the China's Space Station around 2027. The main goal of the experiment is the measurement of cosmic rays up to energies which are not explored by the instruments currently operating in space, in particular protons with energies up to $\mathrm{PeV}$, nuclei up to hundreds of TeV per nucleon and electrons up to tens of TeV. HERD will consist of silicon charge detectors, anti-coincidence scintillators, scintillating fiber trackers, a transition radiation detector and a calorimeter. The latter is a homogeneous, deep, 3D segmented calorimeter made of about 7500 LYSO cubic crystals: thanks to this innovative design, it will achieve large acceptance, good energy resolution and excellent electron/proton discrimination. In order to increase both energy calibration capabilities and redundancy of the instrument, the LYSO scintillation light will be read-out by two independent systems: the first is made of wave-length shifting fibers coupled with imaged intensified CMOS cameras, and the second one consists of photodiodes with different active areas connected to a custom front-end electronics. Both read-out systems are designed to have a large dynamic range, up to $10^{7}$, and a low power consumption. The design of the calorimeter is validated by several Monte Carlo simulations and beam test results obtained with detector prototypes. In this paper we describe the anticipated performances of the calorimeter and the current status of the double read-out system, and we discuss the recent developments of both the HERD prototype and the flight model design.

$37^{\text {th }}$ International Cosmic Ray Conference (ICRC 2021)

July 12 th - 23rd, 2021

Online - Berlin, Germany 


\section{Introduction}

Observations of cosmic ray (CR) fluxes provide unique information regarding several physics phenomena, e.g. the acceleration and propagation of particles inside the Galaxy and the nature of dark matter. Typical simplified models based on experimental results up to 2010 foresee single power law spectra from tens $\mathrm{GeV}$ per nucleon up to the "knee" region (around $10^{15} \mathrm{eV}$ ). Recent CR measurements have shown several unexpected behaviors of charged particle fluxes: e.g. a hardening at around $250 \mathrm{GeV}$ per nucleon of nuclei spectra was observed by several experiments[1][2][3]. A softening of proton spectrum above $10 \mathrm{TeV}$ was also reported[4]. Those results have large impact on our understanding of propagation of CR in the Galaxy; furthermore the observation of cosmic electrons and positrons up to the $\mathrm{TeV}$ region provides information to constrain the properties of nearby astrophysical source and of models for indirect dark matter production.

The High Energy cosmic-Radiation Detection (HERD) is a China-led international space mission in collaboration with several European institutes. The detector will be installed aboard the Chinese Space Station (CSS) around 2027. The main goal of the experiment is to extend the measurement of cosmic ray spectra up to the knee region thanks to a large geometrical factor, which will be one order of magnitude larger than that of current space experiments. The HERD instrument will also be capable of accurate electron+positron (from now on it is indicated as "electron") flux measurement up to tens $\mathrm{TeV}$ and detection of gamma-rays coming from several sources.

\section{The HERD instrument}

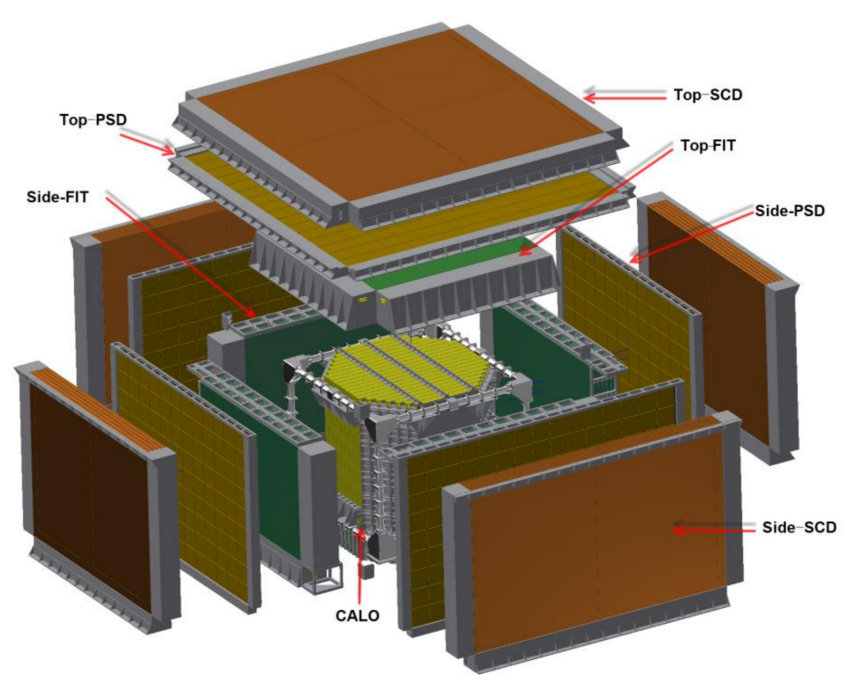

Figure 1: Simplified image of the HERD design.

All operating high energy $\mathrm{CR}$ detectors in orbit can properly measure particles which enter from the top of the detector. In order to increase the geometrical factor, the HERD instrument is designed to accept particles from top and lateral faces of the detector. A simplified design of HERD is shown in fig. 1: starting from the outer layers, Silicon Charge Detectors (SCD), Plastic Scintillator Detectors (PSD), FIber Trackers (FIT) and a Calorimeter (CALO) are present. The 


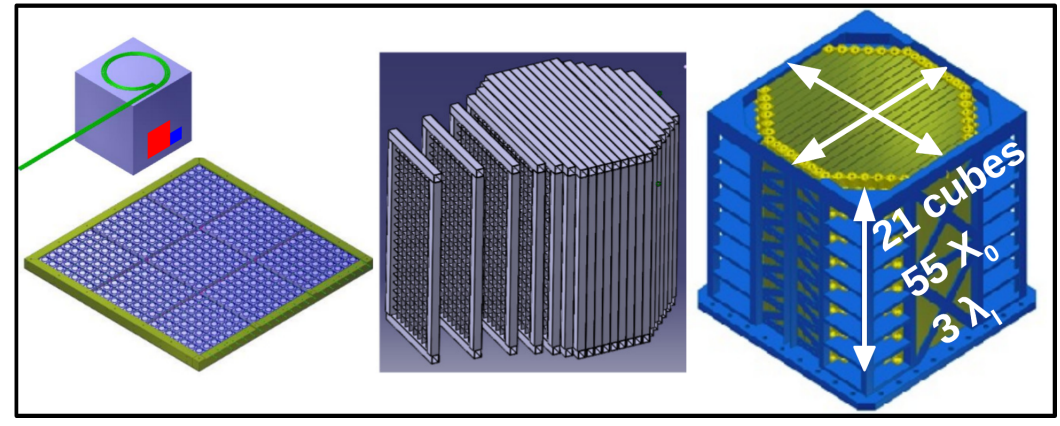

Figure 2: Simplified images of few components of the CALO.

charge of incoming particles is measured by the SCD with several layers of silicon micro-strip detectors. The PSD consists of plastic scintillators read-out by Silicon PhotoMultipliers (SiPM) and it is mainly employed as anti-coincidence detector for the gamma rays analysis. The track of both charged particles and photons is reconstructed with the FIT: it is made of scintillating fibers read-out by SiPM. A Transition Radiation Detector (TRD) (which is not shown in fig. 1) will also be added to a lateral face as the outer detector: it will be calibrated with ground electron beams at the GeV scale and it will be employed during the mission to cross-check the CALO energy scale for protons at $\sim \mathrm{TeV}$.

\section{Design and read-out system of Calorimeter}

The CALO is the main detector of HERD instrument: it is a homogeneous, isotropic, 3D segmented calorimeter which can accepts particles coming from each surface. The performance of a detector based on these ideas was accurately studied by the CaloCube R\&D project[8]. By exploiting those results, the baseline design of the CALO consists of about 7500 LYSO cubes with edge length of $3 \mathrm{~cm}$, corresponding to about 2.6 radiation lengths $\left(\mathrm{X}_{0}\right)$ and 1.4 Molière radius. The CALO external envelope is similar to an octagonal prism and the crystals are arranged on vertical layers with different dimension (fig. 2). The total depth of the CALO for vertical particles is about $55 \mathrm{X}_{0}$ and 3 interaction length $\left(\lambda_{\mathrm{I}}\right)$.

The scintillation light of each crystal is read-out by two independent systems: the first one consists of WaveLength Shifting fibers (WLS) coupled to image Intensified scientific CMOS (IsCMOS) cameras[5], the second one is made of photo-diodes (PD) connected to custom front-end electronics chips named HIDRA[9]. This design, here named "double read-out system", achieves the capability of cross-calibrating the scintillation light measurement. Recent articles by calorimetric CR experiments speculated about the energy scale calibration as a possible source of unknown uncertainty[6][7], particularly for the electron flux measurement. Both the "double read-out" system and the TRD detector will strongly increase the understanding of HERD calorimeter energy scale with respect to previous CR detectors. Furthermore, both WLS and PD systems provide independent fast trigger information which will be employed to improve the HERD trigger capabilities.

WLS-IsCMOS Two WLSs are attached to a face of each cube. Both ends of the each fiber is used to read-out the scintillating light, fig. 3a. Two fiber ends are routed to a high range IsCMOS and a 

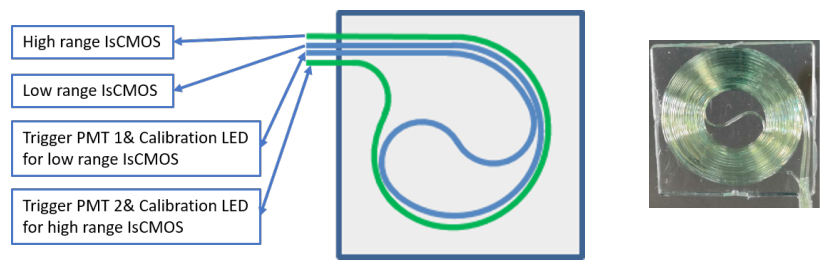

(a) Image and picture of WLS.

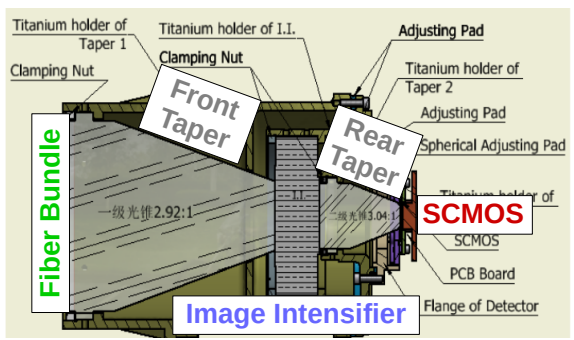

(b) IsCMOS.

Figure 3: 3a shows the design of WLS fibers read-out at both ends and a picture of fiber prototype assembled on a transparent elastometer. $3 \mathrm{~b}$ represents the relevant components of IsCMOS.
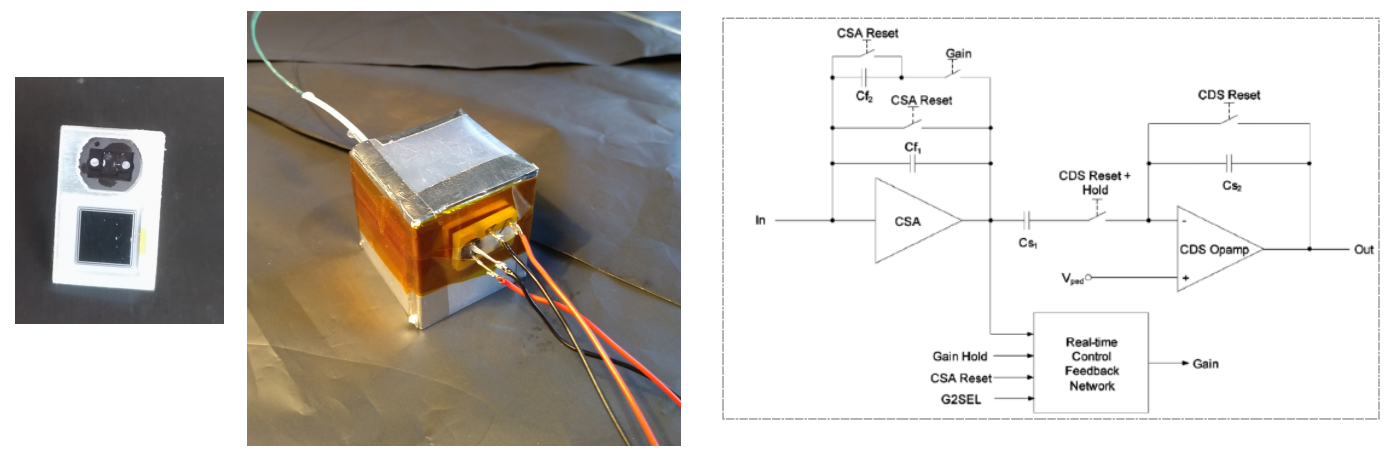

Figure 4: Starting from the left: a preliminary version of the monolithic package including a LPD and a SPD, a LYSO crystal with WLS fibers and PDs, wrapped with an Enhanced Specular Reflector, and a simplified scheme of HIDRA.

low range IsCMOS, while the other ends are connected to a trigger system. IsCMOS consists of an Image Intensifier (II) followed by a scientific CMOS camera, as shown in fig. 3b. The light from WLS fibers is focused on a Micro Channel Plate (MCP) that converts photons into electrons. The latter are converted again into light by a phosphor and it is focused on a CMOS camera by a rear taper. A good material for the phosphor is "P24" since it achieves a high acquisition rate, by having a decay time of about $200 \mu \mathrm{s}$. The CMOS camera is developed by the Xi' an Institute of Optics and Precision Mechanics of the Chinese Academy of Sciences: it is a high frame rate ( $>800$ frames per second), and low read-out noise ( $<1.5$ electrons) image sensor. The pixel side dimension is $40 \mu \mathrm{m}$ and the power consumption is about $40 \mathrm{~W}$ for each camera. About 30 pixels are coupled to the light of a single fiber. By properly selecting the gain of IIs, a good signal noise $(\mathrm{S} / \mathrm{N})$ ratio, of about 10 for MIP signals, and a large dynamic range, of about $10^{7}$, can be achieved.

The fibers dedicated to the trigger systems are grouped in order to give information about the total energy deposit in a specific CALO region. The fiber groups are coupled with an optical system to several Photo Multiplier Tubes (PMT) which provide fast trigger information regarding the specific region. The read-out of both fiber ends allows redundancy inside the trigger system.

PD-HIDRA The configuration of the HERD PD read-out system takes advantage of the results of the CaloCube project[9]. A pair of PDs with different active areas are glued to cubes on the opposite 


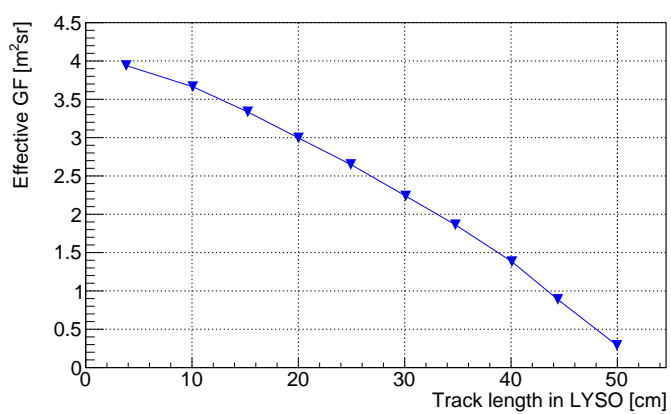

(a) Effective GF

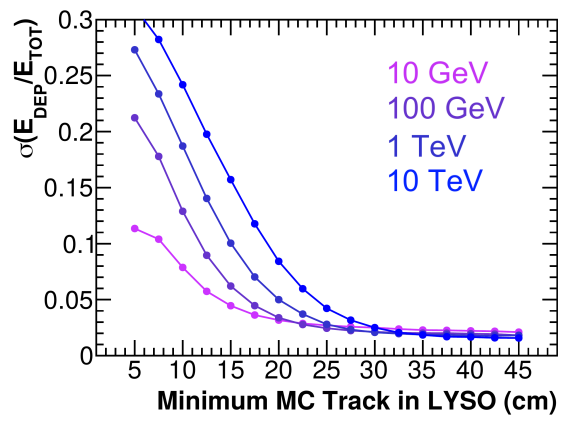

(b) Energy resolution.

Figure 5: CALO performance for electrons obtained with GEANT4 MC simulation. 5a and 5b show the effective geometrical factor for electrons at $1 \mathrm{TeV}$ and the electron energy resolution as a function of the minimum track length inside LYSO crystals respectively.

face with respect the one with WLSs. The sensors are a Large PD (LPD), the VTH2110, and a small one (SPD), the VTP9412, which have an active area of $25 \mathrm{~mm}^{2}$ and $1.6 \mathrm{~mm}^{2}$ respectively. The two sensors are assembled in a single monolithic package: a preliminary version of the package is shown in fig. 4. In addition to the different active areas, for the flight detector, an optical filter with a transmittance of about $1.5 \%$ will be applied on the SPD active area: the ratio between LPD and SPD signals will be of about 1500. The main component of the front-end electronics is the HIDRA chip, developed by INFN for space experiments: it features low noise (about 2500 electrons), low power consumption $(2.8 \mathrm{~mW} / \mathrm{channel}$ ), and large dynamic range (from few fC to $52.6 \mathrm{pC}$ ). A simplified scheme of the chip is reported in fig. 4. The dynamic range is obtained using a charge sensitive amplifier with an automatic double-gain selection and a correlated double sampling circuit: the gain ratio is about 20. Even if the integration window is about $10 \mu \mathrm{s}$, the HIDRA chip provides fast trigger information which can be used as self-trigger for the CALO. LPD and SPD are connected to independent HIDRA channels by using kapton cables developed by INFN. The expected saturation level for the SPD is about $250 \mathrm{TeV}$ per channel, which is enough to measure hadronic showers at $\mathrm{PeV}$ region without sizable degradation of the CALO performance. The SPD S/N ratio is about 10 when scintillation light saturates the corresponding LPD channel: it achieves a large overlap between the two sensors, which is needed for the energy scale calibration. Finally the S/N corresponding to a MIP signal read-out by LPDs is $\geq 4$.

\section{Expected CALO performance: simulations and beam tests}

The innovative CALO design achieves excellent performance for CR measurements. One of the main parameters is the large acceptance: the effective geometrical factor (GF), i.e. the geometrical factor multiplied by the selection efficiency, is larger than $2 \mathrm{~m}^{2} \mathrm{sr}$ for electrons and $1 \mathrm{~m}^{2} \mathrm{sr}$ for protons. Those results are obtained with Monte Carlo (MC) simulations based on GEANT4. A preliminary HERD geometry has been implemented: it includes both active materials and mechanical structures for each sub-detector. Fig 5a shows the effective acceptance for $1 \mathrm{TeV}$ electrons: this result is obtained by employing few minor cuts which select properly developed showers and a 


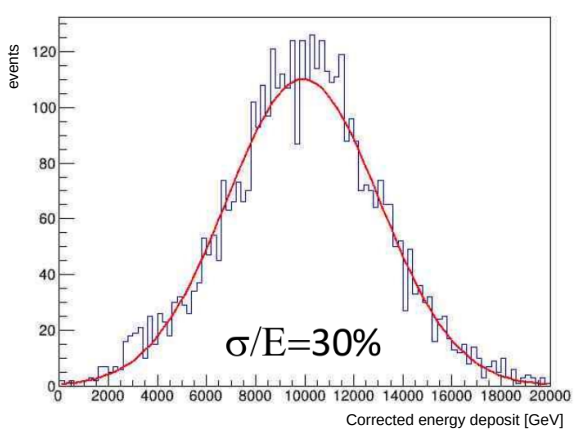

(a) Corrected energy deposit.

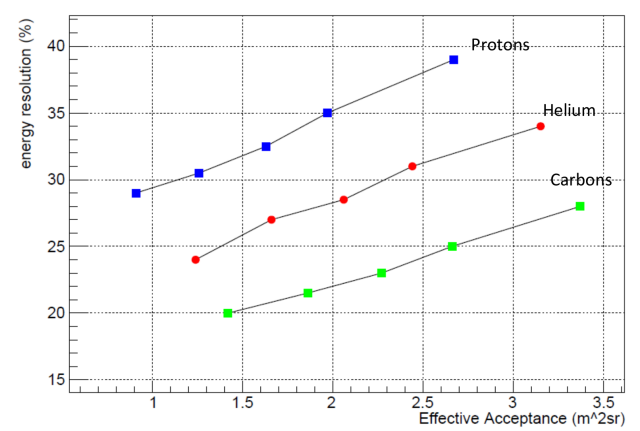

5b: (b) Energy resolution vs effective GFs.

Figure 6: MC simulation results for protons and nuclei. 6a: corrected energy deposit for protons at $1 \mathrm{TeV}$. This correction takes into account the longitudinal leakage and allows the distribution mean to be similar to the primary energy. 6b: energy resolution as a function of the effective GF for $10 \mathrm{TeV}$ hadrons.

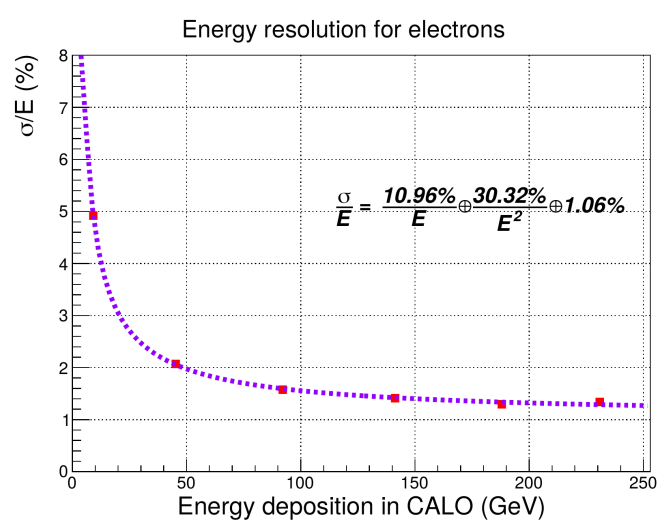

(a) Electron energy resolution with WLS

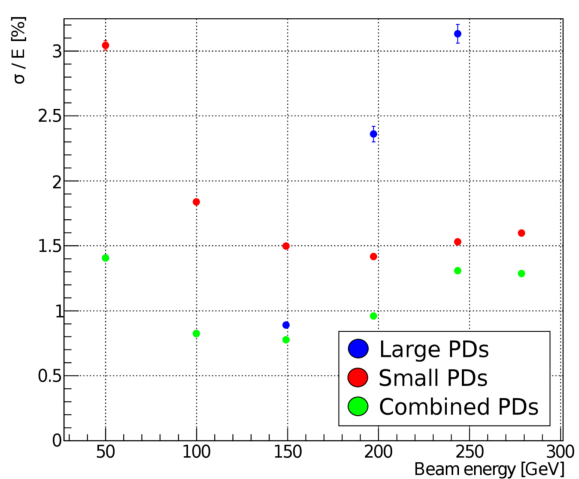

(b) Electron energy resolution with PD

Figure 7: Performance of the read-out systems for electrons. 7a: energy resolution obtained with a prototype made of LYSO cubes read-out WLS-IsCMOS system. 7b: energy resolution obtained by a prototype made of CsI crystals read-out by PD-HIDRA system.

major selection on the minimum length of the primary track (named "minimum track length cut" or MTL) traversed inside the LYSO crystals. For a MTL of about $30 \mathrm{~cm}\left(\sim 26 \mathrm{X}_{0}\right)$ the GF is $>2 \mathrm{~m}^{2} \mathrm{sr}$. For this computation only down-going particles are accepted, to avoid interaction with space station structures. By using these cuts, the energy resolution of electrons up to $10 \mathrm{TeV}$ is $2 \%$ if a MTL of $30 \mathrm{~cm}$ is applied, e.g. fig. 5b. Thanks to the $3 \mathrm{D}$ imaging capabilities, the CALO can also provide independent information on the primary particle track direction. Regarding the electron/proton discrimination power, by using a preliminary version of selection algorithm based on Boosted Decision Tree (contained in TMVA-ROOT package), a residual proton contamination of few percent for high energy electrons is anticipated.

Thanks to the CALO depth, the performance for protons and nuclei is excellent for a space calorimeter. In order to improve the energy resolution for hadronic showers, a correction of the energy deposit based on the longitudinal development of the shower is applied. By applying selections which reject showers with large leakage from the CALO, the effective GF for protons and nuclei up to hundreds 
$\mathrm{TeV}$ is $\geq 1 \mathrm{~m}^{2} \mathrm{sr}$ : the energy resolution is better than $30 \%$. Fig. 6 shows the corrected energy deposit for protons at $1 \mathrm{TeV}$ and the energy resolution as a function of the effective GF at $10 \mathrm{TeV}$; the latter is obtained by applying cuts on the shower length in CALO using different thresholds. In order to validate the CALO performance obtained by MC simulations, tests with high energy beams are done. A prototype made of 5x5x20 LYSO cubes read-out with the WLS-IsCMOS system was tested in 2017 at the CERN SPS. The energy resolution for electrons is shown in fig. 7a: it is better than $2 \%$ above $100 \mathrm{GeV}$ and it is consistent with the MC simulation predictions. The energy deposit and energy resolution of protons obtained with beam test data are also consistent with the MC expected one, better than $30 \%$ at $400 \mathrm{GeV}$. The PD-HIDRA system was tested by the CaloCube collaboration. The energy resolution obtained with a prototype made of $\sim 400 \mathrm{CsI}(\mathrm{Tl})$ crystals is shown in fig. 7b: few LPD channels saturate with electrons above $150 \mathrm{GeV}$. By using combined information of LPD and SPD a energy resolution better than $2 \%$ is confirmed. More details regarding the several beam tests of PD system are reported in: [10][11][12].

\section{Conclusion}

The innovative design of the HERD calorimeter allows a large effective geometrical factor which is about one order of magnitude larger than the one obtained by previous cosmic ray calorimetric experiments. In parallel, a good energy resolution is expected by Monte Carlo simulations and confirmed by beam tests both for leptons and hadrons. Furthermore, the double read-out scheme will strongly improve both the calibration and trigger capabilities, which usually have large impact on the systematic errors of flux measurements. Finally, a new calorimeter prototype including both WLSs and PDs is under construction and will be tested at the CERN SPS in November 2021.

\section{References}

[1] O. Adriani et al., Science 01 Apr 2011: Vol. 332, Issue 6025, pp. 69-72

[2] M. Aguilar et al., PHYSICAL REVIEW LETTERS 114, 171103 (2015)

[3] O. Adriani et al., PHYSICAL REVIEW LETTERS 125, 251102 (2020)

[4] Q. An et al., Science Advances 27 Sep 2019, Vol. 5, no. 9, eaax3793

[5] Y. Dong et al., PoS(ICRC2019) 062

[6] O. Adriani et al., PHYSICAL REVIEW LETTERS 120, 261102 (2018)

[7] DAMPE Collaboration, Nature 552, 63-66 (2017).

[8] O. Adriani et al., Astroparticle Physics 96 (2017) 11-17

[9] O. Adriani et al 2019 JINST 14 P1100

[10] E. Vannuccini et al., NIM A, Volume 845, 11 February 2017, Pages 421-424

[11] R. D’Alessandro et al., NIM A, Volume 824, 11 July 2016, Pages 609-613

[12] L. Pacini et al., J. Phys. Conf. Ser., Volume 928, number 1, pages 012013, 2017 


\section{Full Authors List: HERD Collaboration}

O. Adriani ${ }^{26}$, F. Alemanno ${ }^{27}$, R. Aloisio ${ }^{27}$, C. Altomare ${ }^{23}$, G. Ambrosi ${ }^{34}$, Q. An ${ }^{10}$, M. Antonelli ${ }^{45}$, P. Azzarello ${ }^{37}$, L. Bai ${ }^{8}$, Y.L. Bai ${ }^{3}$, T.W. Bao ${ }^{1}$, M. Barbanera ${ }^{34}$, F.C.T. Barbato ${ }^{27}$, P. Bernardini ${ }^{30}$, E. Berti ${ }^{26}$, B. Bertucci ${ }^{35}$, X.J. Bi ${ }^{1}$, G. Bigongiari ${ }^{36}$, M. Bongi ${ }^{26}$, V. Bonvicini $^{45}$, P. Bordas ${ }^{40}$, V. Bosch-Ramon ${ }^{40}$, S. Bottai ${ }^{25}$, P. Brogi ${ }^{36}$, F. Cadoux ${ }^{37}$, D. Campana ${ }^{31}$, W.W. Cao ${ }^{3}$, Z. Cao ${ }^{1}$, J. Casaus ${ }^{39}$, E. Catanzani ${ }^{35}$, P. W. Cattaneo ${ }^{33}$, J. Chang ${ }^{9,13}$, Y.H. Chang ${ }^{21}$, G.M. Chen ${ }^{1}$, Y. Chen ${ }^{15}$, F. Cianetti ${ }^{35}$, A. Comerma ${ }^{40,41}$, D. Cortis ${ }^{28}$, X.H. Cui ${ }^{13}$, X.Z. Cui ${ }^{1}$, C. Dai ${ }^{5}$, Z.G. Dai ${ }^{15}$, R. D’Alessandro ${ }^{26}$, S. De Gaetanoe ${ }^{24}$, I. De Mitri ${ }^{27}$, F. de Palma ${ }^{30}$, V. Di Felice ${ }^{50}$, A. Di Giovanni ${ }^{27}$, M. Di Santo ${ }^{27}$, L. Di Venere ${ }^{24}$, J.N. Dong ${ }^{6,7}$, Y.W. Dong ${ }^{1}$, G. Donvito ${ }^{23}$, M. Duranti ${ }^{34}$, D. D’Urso ${ }^{49}$, C. Evoli ${ }^{27}$, K. Fang ${ }^{1}$, L. Fariña ${ }^{42}$, Y. Favre ${ }^{37}$, C.Q. Feng ${ }^{10}$, H. Feng ${ }^{16}$, H.B. Feng ${ }^{5}$, Z.K. Feng ${ }^{5}$, N. Finetti ${ }^{22}$, V. Formato ${ }^{50}$, J. M. Frieden ${ }^{44}$, P. Fusco $^{24}$, J.R. Gao ${ }^{3}$, F. Gargano ${ }^{23}$, D. Gascon-Fora ${ }^{40}$, D. Gasparrini ${ }^{50}$, N. Giglietto ${ }^{24}$, F. Giovacchini ${ }^{39}$, S. Gomez ${ }^{40}$, K. Gong ${ }^{1}$, Q.B. $\mathrm{Gou}^{1}$, R. Guida ${ }^{46}$, D.Y. Guo ${ }^{1}$, J.H. Guo ${ }^{9}$, Y.Q. Guo ${ }^{1}$, H.H. He ${ }^{1}$, H.B. Hu${ }^{1}$, J.Y. Hu ${ }^{1,2}$, P. Hu ${ }^{1,2}$, Y.M. Hu ${ }^{9}$, G.S. Huang ${ }^{10}$, J. Huang ${ }^{1}$, W.H. Huang ${ }^{6,7}$, X.T. Huang ${ }^{6,7}$, Y.B. Huang ${ }^{5}$, Y.F. Huang ${ }^{15}$, M. Ionica ${ }^{34}$, L. Jouvin ${ }^{42}$, A. Kotenko ${ }^{37}$, D. Kyratzis ${ }^{27}$, D. La Marra ${ }^{37}$, M.J. Li ${ }^{6,7}$, Q.Y. Li ${ }^{6,7}$, R. Li ${ }^{3}$, S.L. Li ${ }^{1,2}$, T. Li ${ }^{6,7}$, X. Li ${ }^{9}$, Z. Li ${ }^{17}$, Z.H. Li ${ }^{1,2}$, E.W. Liang ${ }^{5}$, M.J. Liang ${ }^{1,2}$, C.L. Liao ${ }^{8}$, F. Licciulli ${ }^{23}$,

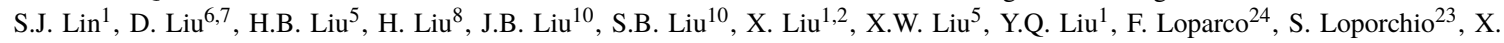
$\mathrm{Lu}^{5}$, J.G. Lyu ${ }^{4}$, L.W. Lyu ${ }^{3}$, P. Maestro ${ }^{36}$, E. Mancini ${ }^{34}$, R. Manera ${ }^{40}$, J. Marin ${ }^{39}$, P. S. Marrocchesi ${ }^{36}$, G. Marsella ${ }^{53}$, G. Martinez ${ }^{39}$, M. Martinez ${ }^{42}$, D. Marzullo ${ }^{47}$, J. Mauricio ${ }^{40}$, E. Mocchiutti ${ }^{45}$, G. Morettini ${ }^{35}$, N. Mori ${ }^{25}$, L. Mussolin ${ }^{35}$, M. Nicola Mazziotta ${ }^{23}$, A. Oliva $^{51}$, D. Orlandi ${ }^{28}$, G. Osteria ${ }^{31}$, L. Pacini ${ }^{25}$, B. Panico ${ }^{31}$, F.R. Pantalei ${ }^{24}$, S. Papa ${ }^{46}$, P. Papini ${ }^{25}$, J.M. Paredes ${ }^{40}$, A. Parenti ${ }^{27}$, M.

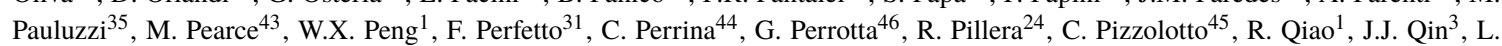
Quadrani $^{51,52}$, Z. Quan ${ }^{1}$, A. Rappoldi ${ }^{33}$, G. Raselli ${ }^{33}$, X.X. Ren ${ }^{6,7}$, F. Renno ${ }^{46}$, M. Ribo ${ }^{40}$, J. Rico ${ }^{42}$, M. Rossella ${ }^{33}$, F. Ryde ${ }^{43}$, A. $\mathrm{Sanmukh}^{40}$, V. Scotti ${ }^{32}$, D. Serini ${ }^{23}$, D.L. Shi ${ }^{3}$, Q.Q. Shi ${ }^{6,7}$, L. Silveri2 ${ }^{27}$, O. Starodubtsev ${ }^{25}$, D.T. Su ${ }^{12}$, M. Su $^{20}$, D. Sukhonos ${ }^{37}$, A. $\mathrm{Suma}^{46}$, X.L. Sun ${ }^{1}$, Z.T. Sun ${ }^{1,2}$, A. Surdo ${ }^{29}$, Z.C. Tang ${ }^{1}$, A. Tiberio ${ }^{26}$, A. Tykhonov ${ }^{37}$, V. Vagelli ${ }^{48}$, E. Vannuccini ${ }^{25}$, M. Velasco ${ }^{39}$, R. Walter ${ }^{38}$, A.Q. Wang ${ }^{6,7}$, B. Wang ${ }^{3}$, J.C. Wang ${ }^{14}$, J.M. Wang ${ }^{1}$, J.J. Wang ${ }^{1,2}$, L. Wang ${ }^{13}$, M. Wang ${ }^{6,7}$, R.J. Wang ${ }^{1}$, S. Wang ${ }^{9}$, X.Y. $\mathrm{Wang}^{15}$, X.L Wang ${ }^{10}$, Z.G. Wang ${ }^{1}$, D.M. Wei ${ }^{9}$, J.J. Wei ${ }^{9}$, B.B. Wu ${ }^{1}$, J. Wu ${ }^{19}$, L.B. Wu ${ }^{27}$, X. Wu ${ }^{37}$, X.F. Wu ${ }^{9}$, Y.L. Xin ${ }^{8}$, M. Xu ${ }^{1}$, Z.Z. $\mathrm{Xu}^{10}$, H.R. Yan ${ }^{17}$, Y. Yang ${ }^{3}$, P.F. Yin ${ }^{1}$, Y.W. Yu ${ }^{18}$, Q. Yuan ${ }^{9}$, G. Zampa ${ }^{45}$, N. Zampa ${ }^{45}$, M. Zha ${ }^{1}$, C. Zhang ${ }^{1}$, F.Z. Zhang ${ }^{1,2}$, L. Zhang ${ }^{1}$, L. Zhang ${ }^{11}$, L.F. Zhang ${ }^{1,2}$, S.N. Zhang ${ }^{1,2}$, Y. Zhang ${ }^{9}$, Y.L. Zhang ${ }^{10}$, Z.G. Zhao ${ }^{10}$, J.K. Zheng ${ }^{3}$, Y.L. Zhou ${ }^{5}$, F.R. Zhu ${ }^{8}$, and K.J. Zhu ${ }^{4}$

1 Key Laboratory of Particle and Astrophysics, Chinese Academy of Sciences, Beijing, China

2 University of Chinese Academy of Sciences, Beijing, China

Xi' an Institute of Optics and Precision Mechanics of CAS, Xi' an, China

Institute of High Energy Physics, Chinese Academy of Sciences, Beijing, China

School of Physical Science and Technology, Guangxi University, Nanning, China

Institute of Frontier and Interdisciplinary Science, Shandong University, Qingdao, China

Key Laboratory of Particle Physics and Particle Irradiation, Ministry of Education, China

School of Physical Science and Technology, Southwest Jiaotong University, Chengdu, China

Key Laboratory of Dark Matter and Space Astronomy, Purple Mountain Observatory, Chinese Academy of Sciences, Nanjing 210023, China

10 Department of Modern Physics, University of Science and Technology of China, Hefei, China

11 Department of Astronomy, Yunan University, Kunming, China

12 North Night Vision Technology co. ltd., Kunming, China

13 National Astronomical Observatories, Chinese Academy of Sciences, Beijing 100101, China

14 Yunnan Astronomical Observatory, Chinese Academy of Sciences, Kunming, China

15 School of Astronomy and Space Science, Nanjing University, Nanjing, China

16 Department of Astronomy, Tsinghua University, Beijing, China

17 Department of Astronomy, Peking University, Beijing, China

18 Institute of Astrophysics, Central China Normal University, Wuhan, China

19 Department of Physics, China University of Geosciences, Wuhan, China

20 The University of Hong Kong, Hong Kong, China

21 Institute of Physics, Academia Sinica, Taipei, Taiwan

22 Universitá dell'Aquila and Istituto Nazionale di Fisica Nucleare, Sezione di Firenze, Firenze, Italy

23 Istituto Nazionale di Fisica Nucleare, Sezione di Bari, Bari, Italy

24 Dipartimento di Fisica "M.Merlin" dell?Univerisitá e del Politecnico di Bari, and Istituto Nazionale di Fisica Nucleare, Sezione di Bari, Bari, Italy

25 Istituto Nazionale di Fisica Nucleare, Sezione di Firenze, Firenze, Italy

26 Universitá di Firenze and Istituto Nazionale di Fisica Nucleare, Sezione di Firenze, Firenze, Italy

27 Gran Sasso Science Institute, L'Aquila, Italy and INFN Laboratori Nazionali del Gran Sasso, Assergi, L’Aquila, Italy

28 INFN Laboratori Nazionali del Gran Sasso, L'Aquila , Italy

29 Istituto Nazionale di Fisica Nucleare, Sezione di Lecce, Lecce, Italy

30 Universitá del Salento and Istituto Nazionale di Fisica Nucleare, Sezione di Lecce, Lecce, Italy

31 Istituto Nazionale di Fisica Nucleare, Sezione di Napoli, Napoli, Italy

32 Universitá di Napoli "Federico II" and Istituto Nazionale di Fisica Nucleare, Sezione di Napoli, Napoli, Italy

33 Istituto Nazionale di Fisica Nucleare, Sezione di Pavia, Pavia, Italy 
34 Istituto Nazionale di Fisica Nucleare, Sezione di Perugia, Perugia, Italy

35 Universitá degli Studi di Perugia and Istituto Nazionale di Fisica Nucleare, Sezione di Perugia, Perugia, Italy

36 Universitá di Siena and Istituto Nazionale di Fisica Nucleare, Sezione di Pisa, Pisa, Italy

37 Département de Physique Nucléaire et Corpusculaire (DPNC), Université de Genève, Genève, Switzerland

38 Department of Astronomy, University of Geneva, Geneva, Switzerland

39 Centro de Investigaciones Energéticas, Medioambientales y Tecnoló gicas (CIEMAT), E-28040 Madrid, Spain

40 Dept. Física Quàntica i Astrofísica, Institut de Ciències del Cosmos (ICCUB), Universitat de Barcelona (IEEC-UB), Barcelona, Spain

41 Universitat Pompeu Fabra (UPF), Barcelona, Spain

42 Institut de Física d'Altes Energies (IFAE), The Barcelona Institute of Science and Technology (BIST), E-08193 Bellaterra, Barcelona, Spain

43 KTH Royal Institute of Technology, Stockholm, Sweden

44 Institute of Physics, Ecole Polytechnique Fédérale de Lausanne (EPFL), Lausanne, Switzerland

45 Istituto Nazionale di Fisica Nucleare, Sezione di Trieste, Trieste, Italy

46 Universitá degli Studi di Napoli Federico II, Napoli, Italy

47 Universitá degli Studi di Trieste, Italy

48 Italian Space Agency and Istituto Nazionale di Fisica Nucleare, Sezione di Perugia, Perugia, Italy

49 Universitá degli Studi di Sassari and Istituto Nazionale di Fisica Nucleare, Sezione di Perugia, Perugia, Italy

50 Istituto Nazionale di Fisica Nucleare, Sezione di Roma Tor Vergata, Rome, Italy

51 INFN Sezione di Bologna, 40126 Bologna, Italy

52 INFN Sezione di Bologna, 40126 Bologna, Italy; Universitá di Bologna, 40126 Bologna, Italy

53 Dipartimento di Fisica e Chimica "E. Segrè" Universitá degli Studi di Palermo, Palermo, Italy and INFN sez. Catania, Catania, Italy 